LA-UR-00-1318

\title{
Generalizing the Heisenberg uncertainty relation
}

\author{
Eric D. Chisolm ${ }^{a}$ \\ T-1, MS B221 \\ Los Alamos National Laboratory \\ Los Alamos, NM 87545
}

February 1, 2008

\begin{abstract}
The proof of the Heisenberg uncertainty relation is modified to produce two improvements: (a) the resulting inequality is stronger because it includes the covariance between the two observables, and (b) the proof lifts certain restrictions on the state to which the relation is applied, increasing its generality. The restrictions necessary for the standard inequality to apply are not widely known, and they are discussed in detail. The classical analog of the Heisenberg relation is also derived, and the two are compared. Finally, the modified relation is used to address the apparent paradox that eigenfunctions of the $z$ component of angular momentum $L_{z}$ do not satisfy the $\phi-L_{z}$ Heisenberg relation; the resolution is that the restrictions mentioned above make the usual inequality inapplicable to these states. The modified relation does apply, however, and it is shown to be consistent with explicit calculations.
\end{abstract}

\section{INTRODUCTION}

The Heisenberg uncertainty relation in its general form for observables $A$ and $B$,

$$
\Delta A \Delta B \geq \frac{1}{2}|i\langle[A, B]\rangle|,
$$


is proved in every intermediate quantum mechanics textbook (and also in the Appendix); its best known special case, $\Delta x \Delta p \geq \frac{\hbar}{2}$, comes from the canonical commutation relation $[x, p]=i \hbar$. A very slight modification of a standard proof of this inequality used by both Bohm ${ }^{1}$ and Sakurai ${ }^{2}$ yields two useful improvements:

1. The resulting inequality is a stronger one that incorporates the covariance between $A$ and $B$, a measure of their statistical correlation. As a bonus, this allows a comparison with the corresponding classical inequality, in which the covariance also appears.

2. This result lifts certain restrictions that must be imposed on the state of the system for the standard Heisenberg inequality to be valid. These restrictions are not generally mentioned in textbooks, but you ignore them at your peril. For example, the $z$ component of angular momentum $L_{z}$ and the azimuthal angle $\phi$ form a canonical pair, so from $\left[\phi, L_{z}\right]=i \hbar$ one expects to find $\Delta \phi \Delta L_{z} \geq \frac{\hbar}{2}$. However, consider the state

$$
\psi(\phi)=\frac{1}{\sqrt{2 \pi}} e^{i m \phi}
$$

This is an eigenstate of $L_{z}$, so $\Delta L_{z}=0$, and a quick calculation yields $\Delta \phi=\frac{\pi}{\sqrt{3}}$, so

$$
\Delta \phi \Delta L_{z}=0<\frac{\hbar}{2}
$$

What went wrong? This example has produced a flurry of commentary over the years ${ }^{3-8}$, and its resolution lies in the surprising fact that eigenstates of $L_{z}$ do not satisfy the criteria necessary for the standard Heisenberg principle to apply. I will describe these criteria in detail below, as well as why eigenstates of $L_{z}$ do not satisfy them, and once I have derived the modified inequality I will show that it is consistent with this example.

The extension to include the covariance is not new ${ }^{9-11}$ (in fact, it was known to Schrödinger ${ }^{12}$ and has been discussed before in this journal ${ }^{13}$ ), nor is the modification that removes certain restrictions on the states ${ }^{14,15}$. However, the proof presented here yields both improvements simultaneously with great ease, and the two together allow one to discuss issues that make it clear that 
quantum mechanics is not a straightforward generalization of classical statistics, even once one has taken into account the noncommutivity of observables. Certain uniquely quantum mechanical concerns require that even the definitions of statistical quantities be made with care, as will be shown below.

\section{THE CLASSICAL UNCERTAINTY RELATION}

Since the modified inequality allows me to compare the Heisenberg relation with its classical counterpart, I will derive the classical relation first. (This relation is also derived in Ref. 13.)

Let $a$ be a classical statistical variable with mean $\langle a\rangle$ and uncertainty $\Delta a$ defined by

$$
(\Delta a)^{2}=\left\langle(a-\langle a\rangle)^{2}\right\rangle=\left\langle a^{2}\right\rangle-\langle a\rangle^{2},
$$

and let $\sigma_{a b}$, the covariance between variables $a$ and $b$, be defined by

$$
\sigma_{a b}=\langle(a-\langle a\rangle)(b-\langle b\rangle)\rangle=\langle a b\rangle-\langle a\rangle\langle b\rangle .
$$

Notice that $(\Delta a)^{2}=\sigma_{a a}$ and that $a$ and $b$ are statistically uncorrelated if and only if $\sigma_{a b}=0$. I define a new variable $\bar{a}$ by $\bar{a}=a-\langle a\rangle$ and similarly for $b$; then $\langle\bar{a}\rangle=\langle\bar{b}\rangle=0$ and

$$
(\Delta a)^{2}=\left\langle\bar{a}^{2}\right\rangle \text { and } \sigma_{a b}=\langle\bar{a} \bar{b}\rangle .
$$

Now I can prove the uncertainty relation. Let $x$ be any statistical variable; then $\left\langle x^{2}\right\rangle \geq 0$ and $\left\langle x^{2}\right\rangle=0$ if and only if $x=0$. Then for the special case $x=\bar{a}+\lambda \bar{b}$ for any $\lambda$ I have

$$
\left\langle x^{2}\right\rangle=\left\langle\bar{a}^{2}\right\rangle+\lambda^{2}\left\langle\bar{b}^{2}\right\rangle+2 \lambda\langle\bar{a} \bar{b}\rangle \geq 0
$$

with equality if and only if $\bar{a}+\lambda \bar{b}=0$. The central expression above is a quadratic in $\lambda$ which according to the inequality has at most one real root (if it had two then it would dip below the $\lambda$-axis and be negative). The condition for the quadratic $A x^{2}+B x+C$ to have at most one real root is $B^{2}-4 A C \leq 0$, with equality in the case of exactly one root. In this case the condition becomes

$$
4\langle\bar{a} \bar{b}\rangle^{2}-4\left\langle\bar{a}^{2}\right\rangle\left\langle\bar{b}^{2}\right\rangle \leq 0
$$

or in terms of (6),

$$
\begin{aligned}
(\Delta a)^{2}(\Delta b)^{2} & \geq\left(\sigma_{a b}\right)^{2} \\
\Delta a \Delta b & \geq\left|\sigma_{a b}\right|
\end{aligned}
$$


with equality if and only if $\bar{a}+\lambda \bar{b}=0$ for some $\lambda$. This is the uncertainty principle for classical statistics.

\section{THE MODIFIED HEISENBERG RELATION}

Now I shall derive the corresponding quantum mechanical result. Let $A$ and $B$ be observables, and let states be denoted by $\psi, \chi$, and so on. The inner product of states $\psi$ and $\chi$ is denoted $\langle\psi, \chi\rangle$, and the norm $\|\psi\|$ is defined by $\|\psi\|=\sqrt{\langle\psi, \psi\rangle}$. Finally, the average of $A$ is defined by $\langle A\rangle=\langle\psi, A \psi\rangle$. (I deliberately avoid Dirac's $\langle\psi|A| \psi\rangle$ because it obscures an important issue; see below.)

The quantum mechanical derivation cannot simply recapitulate the classical derivation with the appropriate letters capitalized for two reasons:

\section{1. $A$ and $B$ might not commute.}

Because of this, the order of the factors in the cross term in the expansion of $\left\langle x^{2}\right\rangle$ should be preserved. The problem of noncommutivity actually rears its head earlier, however, in the very definition of covariance, and I must address that issue first. The classical definition of covariance is symmetric in $a$ and $b\left(\sigma_{a b}=\sigma_{b a}\right)$ because $a$ and $b$ always commute, but if I employed the same definition in the quantum case I would find $\sigma_{A B}=\sigma_{B A}+\langle[A, B]\rangle$. A covariance symmetric in $A$ and $B$ is preferable, and the easiest way to achieve this is to define

$$
\begin{aligned}
\sigma_{A B} & =\frac{1}{2}\langle(A-\langle A\rangle)(B-\langle B\rangle)+(B-\langle B\rangle)(A-\langle A\rangle)\rangle \\
& =\frac{1}{2}\langle A B+B A\rangle-\langle A\rangle\langle B\rangle .
\end{aligned}
$$

Now $\sigma_{A B}=\sigma_{B A}$ and $\sigma_{A A}$ has the same form as before, but this definition suffers from another awkward feature that leads to the second point.

\section{The domains of operators matter.}

The domain of an operator $A$, or $\mathcal{D}(A)$, is the set of all vectors $\psi$ in the system's Hilbert space such that $A \psi$ is also a well-defined member of the Hilbert space. (For more on operators with restricted domains, see Refs. 16, 17, and 18. For some of the consequences for quantum mechanics, see Ref. 19.) There are three main reasons that a given $\psi$ might not be in $\mathcal{D}(A)$ : 
(a) The operating prescription for $A$ is not defined for $\psi$. For example, consider the Hilbert space $L^{2}(R)$ and the momentum operator $p=\frac{\hbar}{i} \frac{d}{d x}$. A necessary condition for $p \psi$ to exist is that $\psi$ is differentiable almost everywhere (being defined almost everywhere is enough to specify a member of $\left.L^{2}(R)\right)$; but to be in $L^{2}(R)$ a function merely has to be square integrable, which does not imply differentiability or even continuity. This restriction, though real, is of little practical interest, however, since it is exceedingly rare in applications to encounter this problem.

(b) The operating prescription is well-defined, but the resulting vector is not in the Hilbert space. For example, again consider $L^{2}(R)$ and the momentum operator $p$, and this time let $\psi(x)=\sqrt{2|x|} e^{-|x|}$. Now this $\psi$ is in $L^{2}(R)$ because it is square integrable (in fact, it is normalized), but its derivative

$$
\psi^{\prime}(x)=\frac{x}{|x|} \frac{e^{-|x|}}{\sqrt{2|x|}}(1-2|x|),
$$

while well-defined everywhere except the origin, is not square integrable. Hence $\psi^{\prime}$ is not in $L^{2}(R)$, so $\psi$ is not in $\mathcal{D}(p)$. (It is known that $\mathcal{D}(p)$ is dense ${ }^{20}$ in $L^{2}(R)$, so any $L^{2}$ function is arbitrarily close to a function in $\mathcal{D}(p)$, and this fact is important for quantum mechanics. Nonetheless, $\mathcal{D}(p)$ is not the whole Hilbert space.)

(c) Sometimes $\mathcal{D}(A)$ is restricted to guarantee that $A$ will be Hermitian. For example, consider the space of $L^{2}$ functions of the polar angle $\phi$ and the operator $L_{z}=\frac{\hbar}{i} \frac{d}{d \phi}$. For any two functions $\psi$ and $\chi$, integration by parts shows that

$$
\left\langle\chi, L_{z} \psi\right\rangle=\left\langle L_{z} \chi, \psi\right\rangle+\frac{\hbar}{i}\left[\chi^{*}(2 \pi) \psi(2 \pi)-\chi^{*}(0) \psi(0)\right] .
$$

Thus $L_{z}$ is Hermitian only if its domain is restricted to functions $\psi$ such that $\psi(2 \pi)=e^{i \alpha} \psi(0)$ for some $\alpha$ (note that strict periodicity is not required). As innocent as this seems, this is the source of all of the problems we encountered above with the usual form of the $\phi-L_{z}$ uncertainty relation, as I will show below. 
This issue is the reason that I avoid Dirac's notation $\langle\chi|A| \psi\rangle$; that expression could mean either $\langle\chi, A \psi\rangle$, which requires that $\psi$ is in $\mathcal{D}(A)$ but leaves $\chi$ unrestricted, or $\langle A \chi, \psi\rangle$ ( $A$ is Hermitian), which reverses the restrictions on $\chi$ and $\psi$. The notation used here, on the other hand, is unambiguous. In the derivation of the uncertainty principle, I must keep track of all of the domain requirements imposed on the states in the proof at each step, because the final result will apply only to those states that satisfy all of the restrictions encountered at every step.

With these concerns in mind, I will now consider the quantum mechanical definitions of $\Delta A$ and $\sigma_{A B}$. One usually defines $\Delta A$ by

$$
(\Delta A)^{2}=\left\langle\psi,(A-\langle A\rangle)^{2} \psi\right\rangle=\left\langle A^{2}\right\rangle-\langle A\rangle^{2},
$$

but notice that this expression is defined only for those states that lie in $\mathcal{D}\left(A^{2}\right)$. (Membership in $\mathcal{D}(A)$ is a prerequisite for membership in $\mathcal{D}\left(A^{2}\right)$.) Now I would certainly like $\Delta A$ to be defined for every state for which $\langle A\rangle$ is defined, so I'd like $\Delta A$ to exist for every state in $\mathcal{D}(A)$. The easiest way to do this is to note that by the Hermiticity of $A$, for all states for which the above definition is valid it is equivalent to

$$
(\Delta A)^{2}=\langle(A-\langle A\rangle) \psi,(A-\langle A\rangle) \psi\rangle=\|(A-\langle A\rangle) \psi\|^{2},
$$

and this expression is defined for every state in $\mathcal{D}(A)$. Hence I take Eq. (14), not Eq. (13), to be my definition for $\Delta A$. Remember that it is equivalent to the old definition whenever the old definition is valid, but the old definition is not valid in every case where I would like it to be.

Now on to $\sigma_{A B}$. The definition suggested above,

$$
\begin{aligned}
\sigma_{A B} & =\frac{1}{2}\langle\psi,[(A-\langle A\rangle)(B-\langle B\rangle)+(B-\langle B\rangle)(A-\langle A\rangle)] \psi\rangle \\
& =\frac{1}{2}\langle\psi,(A B+B A) \psi\rangle-\langle A\rangle\langle B\rangle,
\end{aligned}
$$

requires that both $A B \psi$ and $B A \psi$ exist, or that $\psi$ is in both $\mathcal{D}(A B)$ and $\mathcal{D}(B A)$. However, I would prefer a definition of $\sigma_{A B}$ that made only the weaker requirement that $\psi$ is in both $\mathcal{D}(A)$ and $\mathcal{D}(B)$, not least because I want to relate $\sigma_{A B}$ to $\Delta A$ and $\Delta B$, and the weaker requirement is all that is needed to guarantee their existence. Fortunately, this is easy; the Hermiticity 
of $A$ and $B$ allows me to rewrite the above as

$$
\begin{aligned}
\sigma_{A B} & =\frac{1}{2}\langle(A-\langle A\rangle) \psi,(B-\langle B\rangle) \psi\rangle+\frac{1}{2}\langle(B-\langle B\rangle) \psi,(A-\langle A\rangle) \psi\rangle \\
& =\operatorname{Re}\langle(A-\langle A\rangle) \psi,(B-\langle B\rangle) \psi\rangle \\
& =\operatorname{Re}\langle A \psi, B \psi\rangle-\langle A\rangle\langle B\rangle
\end{aligned}
$$

and this definition is valid on the larger set of states that belong to both $\mathcal{D}(A)$ and $\mathcal{D}(B)$, exactly as desired. Hence I take Eq. (16), not Eq. (15), as the definition of covariance. Again, the two expressions are equivalent whenever both are defined, but the first does not exist in every case where I would like it to be, whereas the second does. Finally, in analogy with the classical case I define $\bar{A}=A-\langle A\rangle$, in terms of which

$$
\Delta A=\|\bar{A} \psi\| \quad \text { and } \quad \sigma_{A B}=\operatorname{Re}\langle\bar{A} \psi, \bar{B} \psi\rangle .
$$

Note that $(\Delta A)^{2}=\sigma_{A A}$, just as in the classical case.

Now for the uncertainty relation. The Cauchy-Schwarz inequality says that for any states $\psi$ and $\chi$,

$$
|\langle\chi, \psi\rangle| \leq\|\chi\|\|\psi\|
$$

Then, using Eq. (17),

$$
\begin{aligned}
\Delta A \Delta B & =\|\bar{A} \psi\|\|\bar{B} \psi\| \\
& \geq|\langle\bar{A} \psi, \bar{B} \psi\rangle| \\
& =\sqrt{(\operatorname{Re}\langle\bar{A} \psi, \bar{B} \psi\rangle)^{2}+(\operatorname{Im}\langle\bar{A} \psi, \bar{B} \psi\rangle)^{2}} \\
& =\sqrt{\sigma_{A B}^{2}+(\operatorname{Im}\langle\bar{A} \psi, \bar{B} \psi\rangle)^{2}} .
\end{aligned}
$$

A little algebra shows that $\operatorname{Im}\langle\bar{A} \psi, \bar{B} \psi\rangle=\operatorname{Im}\langle A \psi, B \psi\rangle$, so the final result is

$$
\Delta A \Delta B \geq \sqrt{\sigma_{A B}^{2}+(\operatorname{Im}\langle A \psi, B \psi\rangle)^{2}} .
$$

This is the modified Heisenberg uncertainty relation.

\section{COMMENTS}

First, note that all of the steps leading to Eq. (20) are valid as long as $\psi$ lies in both $\mathcal{D}(A)$ and $\mathcal{D}(B)$, and consequently so is the final result. Therefore, unlike the usual form of the Heisenberg relation, this inequality is 
guaranteed to hold in all circumstances in which the quantities involved (the uncertainties and covariances) are well-defined; there are no more unpleasant surprises waiting to be discovered.

Next, I shall recover the uncertainty relation with which we are familiar. If $\psi$ lies in both $\mathcal{D}(A B)$ and $\mathcal{D}(B A)$, then the following manipulations are allowed:

$$
\begin{aligned}
\operatorname{Im}\langle A \psi, B \psi\rangle & =-\frac{i}{2}\langle A \psi, B \psi\rangle+\frac{i}{2}\langle B \psi, A \psi\rangle \\
& =-\frac{i}{2}\langle\psi, A B \psi\rangle+\frac{i}{2}\langle\psi, B A \psi\rangle \\
& =-\frac{i}{2}\langle\psi,(A B-B A) \psi\rangle \\
& =-\frac{i}{2}\langle[A, B]\rangle .
\end{aligned}
$$

Thus when this additional condition is satisfied,

$$
\Delta A \Delta B \geq \sqrt{\sigma_{A B}^{2}+\frac{1}{4}(i\langle[A, B]\rangle)^{2}},
$$

which implies the standard Heisenberg inequality.

Comparing Eq. (9) with either (20) or (22), we see that the sole difference introduced by quantum mechanics is the term $\operatorname{Im}\langle A \psi, B \psi\rangle$, which on a fairly large class of states is essentially half the expectation value of $i$ times the commutator $[A, B]$. This is the irreducible indeterminacy present even in states where the two observables are entirely independent statistically.

Now I can reconsider the example of the $\phi-L_{z}$ uncertainty relation discussed at the beginning. For the commutator form of the inequality to apply, $\psi$ must lie in the domains of both $\phi L_{z}$ and $L_{z} \phi$, and $\psi=(2 \pi)^{-1 / 2} \exp (i m \phi)$ does not satisfy the latter criterion. If it did, then that would mean that $\phi \psi$ would be in the domain of $L_{z}$, but as I noted earlier every state in the domain of $L_{z}$ must satisfy $\psi(2 \pi)=e^{i \alpha} \psi(0)$, and

$$
\phi \psi(\phi)=\frac{\phi}{\sqrt{2 \pi}} e^{i m \phi}
$$

vanishes at $\phi=0$ and is nonvanishing at $\phi=2 \pi$. Hence $\left(L_{z} \phi\right) \psi$ does not exist, and the commutator inequality does not apply. However, Eq. (20) does 
apply, and to find it for this special case I calculate

$$
\begin{aligned}
\operatorname{Im}\left\langle\phi \psi, L_{z} \psi\right\rangle & =-\frac{i}{2}\left\langle\phi \psi, L_{z} \psi\right\rangle+\frac{i}{2}\left\langle L_{z} \psi, \phi \psi\right\rangle \\
& =-\frac{\hbar}{2} \int_{0}^{2 \pi} \phi \psi^{*} \frac{d \psi}{d \phi} d \phi-\frac{\hbar}{2} \int_{0}^{2 \pi} \frac{d \psi^{*}}{d \phi} \phi \psi d \phi \\
& =-\frac{\hbar}{2} \int_{0}^{2 \pi} \phi\left(\psi^{*} \frac{d \psi}{d \phi}+\psi \frac{d \psi^{*}}{d \phi}\right) d \phi \\
& =-\frac{\hbar}{2} \int_{0}^{2 \pi} \phi \frac{d}{d \phi}\left(\psi^{*} \psi\right) d \phi \\
& =-\frac{\hbar}{2}\left[\phi \psi^{*} \psi\right]_{0}^{2 \pi}+\frac{\hbar}{2} \int_{0}^{2 \pi} \psi^{*} \psi d \phi \\
& =\frac{\hbar}{2}\left(1-2 \pi|\psi(2 \pi)|^{2}\right) .
\end{aligned}
$$

Thus

$$
\Delta \phi \Delta L_{z} \geq \sqrt{\sigma_{\phi L_{z}}^{2}+\frac{\hbar^{2}}{4}\left(1-2 \pi|\psi(2 \pi)|^{2}\right)^{2}} .
$$

For the particular $\psi$ in question, $\sigma_{\phi L_{z}}=0$ (again because $\psi$ is an eigenstate of $L_{z}$ ) and $|\psi(2 \pi)|^{2}=(2 \pi)^{-1}$, so

$$
\Delta \phi \Delta L_{z} \geq 0
$$

which is consistent with what we found at the beginning.

Incidentally, if one carried out an analogous derivation with $x$ and $p$ in place of $\phi$ and $L_{z}$, one would find

$$
\operatorname{Im}\langle x \psi, p \psi\rangle=\frac{\hbar}{2}\left(1-\left[x \psi^{*} \psi\right]_{-\infty}^{\infty}\right)
$$

so the usual Heisenberg inequality for $x$ and $p$ is valid as long as $\psi$ falls off faster than $|x|^{-1 / 2}$ as $|x| \rightarrow \infty$. Since $\psi$ is differentiable almost everywhere it must fall off smoothly, in which case square integrability imposes the above requirement automatically. Hence the standard form of the Heisenberg inequality is always valid for $x$ and $p$. It is precisely the fact that the coordinate $\phi$ is bounded while $x$ is unbounded that allows the sorts of problems considered in this paper to crop up often in one case and not at all in the other.

One final note is in order concerning the $\phi-L_{z}$ inequality. In its current form, Eq. (25), the inequality is not invariant under rotations, as one would 
prefer, since the direction corresponding to $\phi=0$ has no physical significance. (The fact that one must choose a $\phi=0$ direction just to define $\phi$ is the source of the problem.) Hence the $\phi-L_{z}$ inequality has still not been brought to a quite satisfactory form; to finish the job, one must develop rotation-invariant definitions of uncertainty and repeat the proof, which has been done in Ref. 4 .

\section{APPENDIX: ANOTHER STANDARD PROOF OF THE HEISEN- BERG RELATION}

This proof of the uncertainty relation is found, for example, in Ref. 21. Let $A$ and $B$ be observables, let $\psi$ be a state in both $\mathcal{D}(A B)$ and $\mathcal{D}(B A)$ (and thus in $\mathcal{D}(A)$ and $\mathcal{D}(B)$ ), and let $\bar{A}$ and $\bar{B}$ be defined as earlier. Then for any real $\lambda$

$$
\begin{aligned}
\|(\bar{A}+i \lambda \bar{B}) \psi\|^{2} & \geq 0 \\
\langle\psi,(\bar{A}-i \lambda \bar{B})(\bar{A}+i \lambda \bar{B}) \psi\rangle & \geq 0 \\
\left\langle\psi,\left(\bar{A}^{2}+\lambda^{2} \bar{B}^{2}+i \lambda[\bar{A} \bar{B}-\bar{B} \bar{A}]\right) \psi\right\rangle & \geq 0 \\
(\Delta A)^{2}+\lambda^{2}(\Delta B)^{2}+i \lambda\langle[A, B]\rangle & \geq 0
\end{aligned}
$$

where the last line used the standard quantum mechanical definition of uncertainty and the fact that $[\bar{A}, \bar{B}]=[A, B]$. The commutator of two observables is anti-Hermitian, so the quantity $i\langle[A, B]\rangle$ is real. Again we have a quadratic in $\lambda$ with at most one real root, so the same condition as mentioned in the text yields

$$
(i\langle[A, B]\rangle)^{2}-4(\Delta A)^{2}(\Delta B)^{2} \leq 0
$$

or

$$
\Delta A \Delta B \geq \frac{1}{2}|i\langle[A, B]\rangle| .
$$

This is the standard Heisenberg uncertainty relation. This result can be strengthened by replacing $i \lambda$ with $\lambda e^{i \theta}$, treating $\lambda$ as before, and taking the maximum over all $\theta$; the result is Eq. (22). If one modifies this derivation to take into account the new definitions of $\Delta A$ and $\sigma_{A B}$, Eq. (17), one recovers the main result of this paper, Eq. (20). The derivation in Sec. III is much shorter, however.

a) Electronic mail: echisolm@lanl.gov 
1. D. Bohm, Quantum Theory (Prentice-Hall, Englewood Cliffs, NJ, 1951), pp. 205-207.

2. J. J. Sakurai, Modern Quantum Mechanics rev. ed. (Addison-Wesley, New York, 1994), pp. 34-36.

3. D. Judge, "On the uncertainty relation for angle variables," Nuovo Cimento 31 (1964), pp. 332-340.

4. K. Kraus, "Remark on the uncertainty between angle and angular momentum," Z. Phys. 188 (1965), pp. 374-377.

5. P. Carruthers and M. M. Nieto, "Phase and angle variables in quantum mechanics," Rev. Mod. Phys. 40 (1968), pp. 411-440.

6. J. M. Levy-Leblond, "Who is afraid of nonhermitian operators? A quantum description of angle and phase," Ann. Phys. NY 101 (1976), 319341.

7. F. Gesztesy and L. Pittner, "Uncertainty relations and quadratic forms." J. Phys. A 11 (1978), pp. 1765-1770.

8. A. Galindo and P. Pascual, Quantum Mechanics (Springer-Verlag, New York, 1990), vol. 1, pp. 201-206.

9. C. W. Gardiner, Quantum Noise (Springer-Verlag, New York, 1991), pp. $1-2$.

10. V. V. Dodonov, E. V. Kurmyshev, and V. I. Man'ko, "Generalized Uncertainty Relation and Correlated Coherent States," Phys. Lett. A 79 (1980), pp. 150-152. This paper also shows that the uncertainty relation with covariance is valid for mixed states as well.

11. V. V. Dodonov and V. I. Man'ko, "Generalization of the Uncertainity Relations in Quantum Mechanics," in Invariants and the Evolution of Nonstationary Quantum Systems, Vol. 183 of the Proceedings of the Lebedev Physics Institute, ed. M. A. Markov (Nova Science, Commack, NY, 1989), pp. 3-101. This article also discusses the $\phi-L_{z}$ uncertainty relation as well as higher-order uncertainty relations, relations among an arbitrary number of observables, and entropy-based uncertainty relations, and it has a substantial list of references. 
12. E. Schrödinger, "Zum Heisenbergschen unschärfeprinzip," Sitzungsber. K. Preuss. Akad. Wiss. (1930), pp. 296-303.

13. J. Peslak, Jr., "Comparison of classical and quantum mechanical uncertainties," Am. J. Phys. 47 (1979), pp. 39-45.

14. F. Gieres, "Dirac's formalism and mathematical surprises in quantum mechanics," quant-ph/9907069, pp. 21-22, 26-28.

15. See Refs. 4 and 7.

16. N. I. Akhiezer and I. M. Glazman, Theory of Linear Operators in Hilbert Space (Ungar, New York, 1961 and 1963), 2 vol.

17. N. Dunford and J. T. Schwartz, Linear Operators (Interscience, New York, 1958, 1963, and 1971), 3 vol.

18. F. Riesz and B. Sz.-Nagy, Functional Analysis (Ungar, New York, 1955).

19. J. M. Jauch, Foundations of Quantum Mechanics (Addison-Wesley, New York, 1968).

20. See Ref. 19, p. 43.

21. C. Cohen-Tannoudji, B. Diu, and F. Laloë, Quantum Mechanics (John Wiley and Sons, New York, 1977), Vol. 1, pp. 286-287. 